\title{
Making Visible the Invisible
}

Commercial Benefits-Spinoffs

$\mathrm{H}$ ydrogen is a clean-burning gas. Therefore, its flame is nearly invisible during daylight hours. A new system allows technicians to visually spot the invisible, making it possible to shut down the hydrogen source and extinguish a hydrogen fire.

Through NASA Small Business Innovation Research (SBIR) funds at the Stennis Space Center, Duncan Technologies, Inc. (DTI), of Auburn, California, has developed an infrared imaging system that provides color images of invisible hydrogen fires.

Older black-and-white infrared systems have been in use to detect hydrogen fires. However, this type of instrument has a tough time contrasting hydrogen fires from the surrounding background. Often the flame is displayed larger than it actually is, making it less useful in determining which nearby equipment and systems might actually be in danger.

At Stennis, the need for better tools to capture invisible hydrogen fires is mandatory. This NASA field center is the world's largest user of liquid hydrogen, consuming and storing great amounts of the propellant for testing Space Shuttle Main

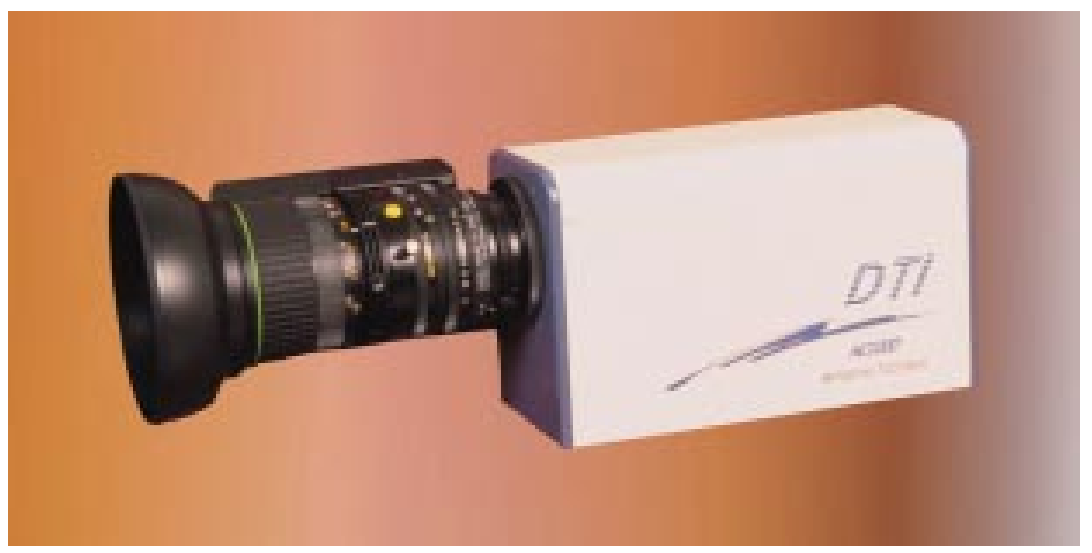

Duncan Technologies, Inc.'s HC1000 uses three different sensors to visually detect a hydrogen flame.
Engines, as well as other rocket propulsion systems and components.

DTI was aware of research carried out at Stennis utilizing images in the infrared and near infrared to view hydrogen fires. DTI's proficiency in advanced imaging and electro-optical services gave the firm an upper hand in identifying the promise of alternate approaches for a hydrogen fire detection system. The infrared sensor technology is very expensive, resolution is poor, and because infrared sensors detect thermal phenomena rather than visible images, the resulting image can be difficult to interpret. By way of an SBIR award, the company looked to perhaps enhance and improve this type of imaging.

DTI built the HC1000, a system that uses three different sensors to visually detect the flame. Two of the sensors operate in the near infrared, while the other sensor works in the visible portion of the electromagnetic spectrum. Of the two near-infrared sensors, one is centered at a spectral band that detects strong water vapor emissions from the flame, while the other is at a band that detects minimal emissions to measure just the background image.

The flame is detected by subtracting the background image from the flame image and filtering the result. This process proved successful. A hydrogen flame can be isolated, then superimposed onto a color video image. A hydrogen flame of just 3 inches is detectable from 150 feet away.

The hydrogen camera was sold to NASA for use at Stennis. In addition, the system was sold to a private automaker to use on test stands, where work on hydrogen-based automobile engines is performed.

DTI developed a marketing plan to commercialize the multispectral imaging camera developed for Stennis. Presently, DTI is introducing a full line of multispectral cameras that enable new capabilities in machine vision, remote sensing, and surveillance applications. The camera products have generated worldwide interest for use in food processing, lumber grading, and quality control of pharmaceuticals. High quality color-infrared imaging for precision agriculture is yet another application.

"All of the multispectral cameras we are currently marketing are a direct spinoff of the hydrogen fire project," says Judy Duncan, DTI vice president. While the market for hydrogen fire detection equipment proved too narrow to support a commercial venture, Duncan adds that by generalizing the technology for industrial and agricultural applications, the marketing of advanced imaging technologies has proven quite successful.

"We invested company funds into continued development and refinement of the system and came up with the current line of products," Duncan says. "At present, we have many potential customers waiting for acquisition of an evaluation unit, which will hopefully be followed by order placement," she concludes. 